\title{
Negative Index in Chiral Metamaterials
}

\author{
Ranjan Singh, ${ }^{1,2, a)}$ Eric Plum, ${ }^{3}$ Christoph Menzel, ${ }^{4}$ Carsten Rockstuhl, ${ }^{4}$ \\ Nikolay I. Zheludev, ${ }^{3}$ and Weili Zhang ${ }^{1, b)}$ \\ ${ }^{1}$ School of Electrical and Computer Engineering, Oklahoma State University, Stillwater, Oklahoma 74078, USA \\ ${ }^{2}$ MPA-CINT, Los Alamos National Laboratory, Los Alamos, NM 87545 \\ ${ }^{3}$ Optoelectronics Research Centre, University of Southampton, SO17 1BJ, UK \\ ${ }^{4}$ Institute of Condensed Matter Theory and Solid State Optics, \\ Friedrich Schiller University Jena, Jena 07743, Germany \\ E-mail:a)ranjan@lanl.gov ; b)weili.zhang@okstate.edu
}

\begin{abstract}
We demonstrate that planar metamaterial lacking of mirror symmetry shows asymmetric transmission of terahertz waves and bands of positive, negative and zero phase and group velocities indicating a polarization sensitive negative index and slow-light media.
\end{abstract}

\section{Introduction}

Metamaterials are composed of periodically arranged artificially designed structures having peculiar properties and promising applications such as negative refraction, superlensing, compact antennas, sensors and slow light devices. Chiral metamaterials are a new class of metamaterials that offer a simple route for achieving negative refraction, tunable circular dichroism and optical activity. A chiral medium is composed of particles that cannot be superimposed on their mirror images and it has different responses for a left circularly polarized (LCP) wave and a right circularly polarized (RCP) wave due to the intrinsic asymmetry of the medium. Planar chiral patterns have an intriguing property that their sense of twist is reversed for observation from opposite sides [1,2]. In this work, we show that planar terahertz chiral metamaterials can show different levels of total transmission for circularly polarized waves of the same handedness propagating in opposite directions. The device also shows bands of positive, negative and zero phase and group velocities indicating a polarization sensitive negative index and slow-light media [3].

\section{Experiment and simulations}

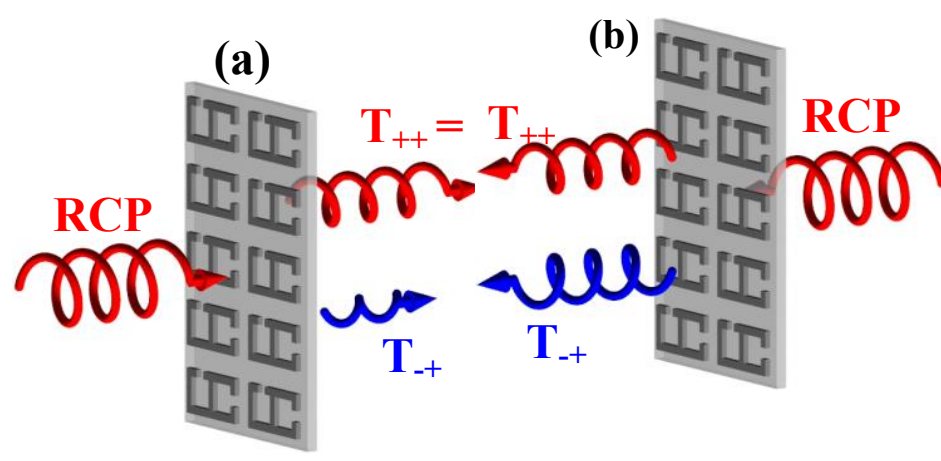

Fig. 1. Asymmetric total transmission of a circularly polarized wave incident on (a) front (b) back side of planar chiral metamaterial. Measured transmission spectra for circularly polarized $\mathrm{THz}$ waves incident on (c) front and (d) back of the metamaterial.

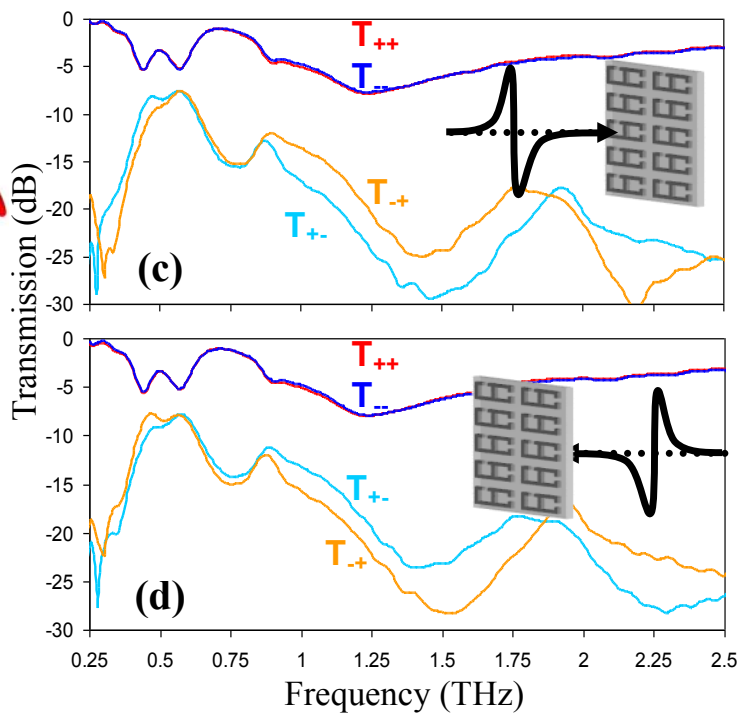

As seen from the schematic in Figs.1a and 1b, and the THz-TDS measurement in Figs. 1c and 1d, the right-to-left T. + and left-to-right $\mathrm{T}_{+}$circular polarization conversion levels depend on both the direction of wave propagation and the handedness of the incident wave, indicating the presence of the asymmetric transmission effect. Importantly counter-propagating circularly polarized waves of the same handedness experience different levels of circular polarization conversion, while their direct transmission levels are identical, for example $\vec{T}_{-+} \neq \bar{T}_{-+}$and $\vec{T}_{++}=\bar{T}_{++}$in case of RCP. It follows that the metamaterial's total transmission for RCP, defined as $T_{+}=T_{++}+T_{-+}$, is asymmetric 
with respect to opposite directions of wave propagation. Moreover, the conversion efficiencies for RCP and LCP are simply switched for opposite directions of wave propagation, i.e. $\vec{T}_{+-}=\bar{T}_{-+}$.

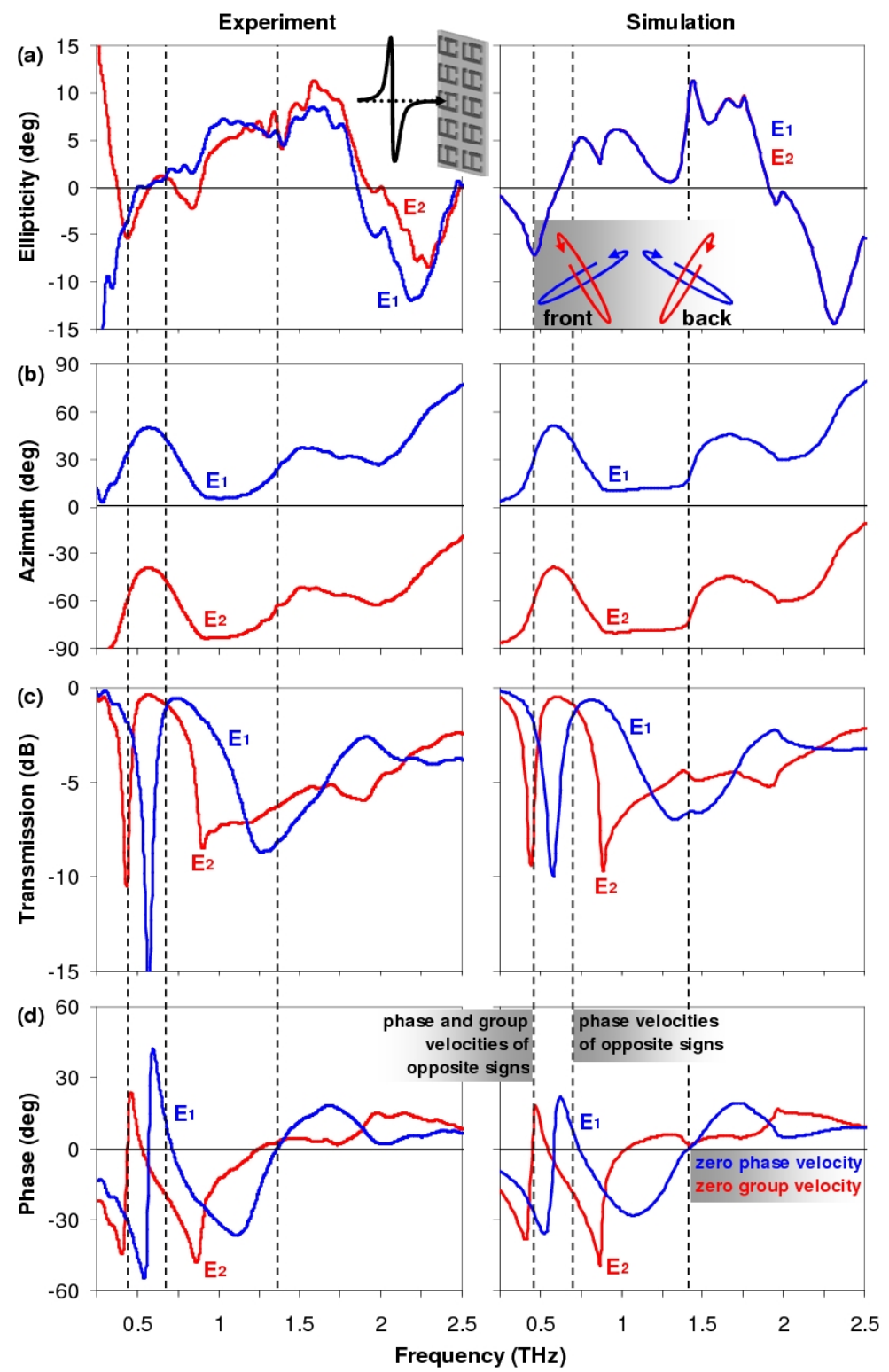

Fig. 2. Transmission eigenstates $E 1$ and $E 2$ for forward propagation in terms of (a) ellipticity angle and (b) azimuth. (c) Transmission level and (d) phase delay for these eigen polarizations.

Ellipticity and azimuth of the transmission eigenstates for waves incident on the front are shown in Figs. $2 \mathrm{a}$ and $2 \mathrm{~b}$. Figures $2 \mathrm{c}$ and $2 \mathrm{~d}$ illustrate the metamaterial transmission properties for its eigen polarizations in terms of transmission levels and phase delay. Interestingly, the metamaterial pattern can introduce positive as well as negative phase delays, indicating that positive and negative phase velocities should be expected in a bulk material based on the structure. These results indicate an opportunity to develop polarization sensitive negative index and slow light media for elliptically polarized waves on the basis of bulk 2D-chiral anisotropic metamaterials.

\section{References}

[1] V. A. Fedotov, P. L. Mladyonov, S. L. Prosvirnin, A. Rogacheva, Y. Chen, and N. I. Zheludev, Phys. Rev. Lett. 97, 167401 (2006).

[2] S. Zhang, Y.-S. Park, J. Li, X. Lu, W. Zhang, and X. Zhang, Phys. Rev. Lett. 102, 023901 (2009).

[3] R. Singh, E. Plum, C. Menzel, C. Rockstuhl, A. K. Azad, R. A. Cheville, F. Lederer, W. Zhang, and N. I. Zheludev, Phys. Rev. B 80, 153104 (2009). 